\title{
Prospecção de Pesquisas sobre o uso de Tecnologias Digitais para o Desenvolvimento do Pensamento Geométrico Espacial
}

\author{
Larissa Weyh Monzon - PPGIE/UFRGS - larissamonzon@ gmail.com \\ Marcus Vinicius de Azevedo Basso - PPGIE/UFRGS - mbasso@ufrgs.br
}

Resumo: Este artigo faz parte de uma pesquisa em andamento na qual se investiga possibilidades de impacto das tecnologias digitais sobre o pensamento geométrico espacial de estudantes do Ensino Básico. Para isso, catalogamos e analisamos, via revisão sistemática da literatura, nove periódicos dos quais registramos a ferramenta tecnológica, os aportes teóricos, a metodologia e as conclusões sobre a temática da investigação. Constatou-se a existência de estudos nas quais tecnologias digitais são apontadas como favorecendo a visualização espacial e, assim, podendo desencadear pensamentos geométricos. Como resultado, identificou-se a necessidade de desenvolver propostas de atividades que contribuam para provocar desequilíbrios cognitivos referentes ao pensamento geométrico espacial de estudantes do ensino básico para proporcionar construções de novos conhecimentos.

Palavras-chave: tecnologias digitais, pensamento geométrico espacial, visualização espacial, revisão sistemática da literatura

\section{Prospecting of researches on the use of Digital Technologies for the Development of Spatial Geometric Thinking}

\begin{abstract}
This article is part of an ongoing research that investigates possibilities of impact of digital technologies on spatial geometric thinking of Basic Education students. For this, we have cataloged and analyzed, through a systematic review of the literature, nine periodicals of which we have registered the technological tool, the theoretical contributions, the methodology and the conclusions about the research theme. It was verified the existence of studies in which digital technologies are pointed as favoring the spatial visualization and, thus, being able to trigger geometric thoughts. As a result, we identified the need of developing proposals for activities that contribute to induce cognitive imbalances related to the spatial geometric thinking of elementary students to provide constructions of new knowledge.
\end{abstract}

Key words: digital tchnologies, spatial geometric thinking, spatial visualization, systematic review of the literature

\section{Introdução}

De acordo com Coll e Monereo (2010), estamos na Sociedade da Informação, que possui uma nova forma de se organizar, de trabalhar, de se comunicar, de relacionar-se, de um modo geral, de viver. As notícias, as informações, os conhecimentos estão sendo transmitidos em tempo real e em quantidades massivas.

Nesta Sociedade da Informação, os estudantes têm acesso e podem apropriar-se das muitas informações disponíveis. Portanto, nesse momento, as escolas estão responsáveis por capacitá-los em organizar, atribuir e dar sentido a esse aglomerado de informações de fácil acesso. 
Tais fatos nos fazem ter a consciência de que o ensino precisa mudar, a sociedade precisa de cidadãos pensantes, autônomos, que construam sua própria visão de mundo, sabendo escolher os critérios mais relevantes e utilizá-los para o seu próprio desenvolvimento e da humanidade.

Em relação à matemática, Tall (1998), diz que as tecnologias realçam a diferença entre as habilidades matemáticas padronizadas e o pensar matematicamente. As máquinas estão fazendo as contas, isso significa que a matemática necessária para os cidadãos da Sociedade da Informação é diferente.

Os recursos digitais estão colocando à disposição dos estudantes mecanismos que favorecem a construção de conceitos e o pensar em matemática, a possibilidade de manipular e acessar objetos matemáticos antes inacessíveis sem as tecnologias (Basso; Notare, 2015). Os computadores estão possibilitando uma nova abordagem da matemática a ser construída na escola, onde novas habilidades podem ser desenvolvidas.

Em se tratando de recursos digitais que disponibilizam visualização, manipulação e criação de sólidos, que estão relacionados às habilidades da geometria espacial, temos a nossa disposição uma variedade de recursos. O ensino de geometria com esses recursos pode proporcionar aos alunos experiências com objetos geométricos complexos, inacessíveis sem as tecnologias, e oferecendo possibilidades de provocar desequilíbrios nas estruturas mentais (Piaget, 1977). Diante disso, observamos a necessidade de investigar como esses recursos podem ser utilizados para promover o desenvolvimento do pensamento geométrico, ou seja, se tecnologias digitais que disponibilizam diferentes tipos de experiências geométricas podem desencadear ações e coordenações de ações que possibilite o sujeito desenvolver novos conceitos e habilidades.

Diante do exposto, e da importância da temática, percebeu-se a necessidade de organizar uma revisão sistemática de literatura dos trabalhos publicados em periódicos encontrados em diferentes bases de dados internacionais e nacionais. Esta busca pretendeu quantificar e analisar os trabalhos que tratavam o ensino da Geometria Espacial com a Tecnologia, para assim realizar um mapeamento, delineando a possibilidade e a viabilidade de trabalhos futuros. A organização deste artigo se deu em seis seções: a primeira seção contextualiza o trabalho; a segunda seção, estuda o desenvolvimento do pensamento geométrico espacial; a terceira seção aborda as potencialidades das tecnologias; na quarta, apresenta os métodos utilizados para a revisão sistemática; na quinta, são apresentados os resultados e discussões das pesquisas encontradas e, na sexta e última seção, as considerações finais e perspectivas de trabalhos futuros.

\section{Pensamento geométrico espacial}

Segundo Oliveira (2005) existem os seguintes tipos de espaços: o matemático, o físico e o psicológico. "Os espaços matemáticos são construídos a partir de axiomas e descritos por uma geometria; algumas dessas geometrias podem definir o espaço físico e o psicológico" (Oliveira, 2005, p. 111). Para Piaget e Inhelder (1981) o espaço psicológico inicia-se fisicamente e matematicamente, dependendo tanto do objeto quanto do sujeito. O sujeito para perceber o mundo físico ao seu redor, o espaço, utiliza seus receptores sensoriais, principalmente o visual e o tátil-cinestésico e para descrever os diferentes tipos de espaços recorre às suas estruturas mentais.

A intuição espacial, ou seja, o reconhecimento do espaço surge no início da existência como um espaço sensório-motor ligado à percepção e à motricidade. Após vem o espaço representativo associado ao início da imagem e do pensamento intuitivo concomitante com a aparição da linguagem, esses espaços são construídos por ações da criança. 
O espaço sensório-motor, movido pela percepção, progride pelas coordenações de ações da criança e seus deslocamentos, a equilibração se dá no nível das ações, ou seja, é um espaço prático. Por volta dos dois anos de idade, com o início da função simbólica, surge o espaço representativo, ou seja, a construção da imagem.

No estádio das operações concretas as ações são mentalizadas tornando coerentes e precisas fazendo a imagem tornar-se independente, já nas operações formais a imagem fica ultrapassada pelo pensamento. Em relação as operações espaciais, a imagem fica cada vez mais secundária a medida que o pensamento evolui, mas permanece por todo o desenvolvimento.

Para que perceba o espaço total, é necessário a noção de distâncias e pontos de vistas, construídos através de dois sistemas: de coordenadas, que permitirá o sujeito a situar os objetos uns em relação aos outros e de um sistema de perspectivas, no qual irá organizar um sistema de referência. A junção desses dois sistemas, que se tratam de um prolongamento das relações topológicas, irá estruturar as três dimensões do espaço euclidiano, tudo isso construído por ações próprias do sujeito.

Para Soto (2012) quando a visualização se refere ao uso da visão para se obter informações em relação à construção de ideias, se estabelece que além de "ver" devemos "saber". A visualização não é um processo de conhecimento direto, pois os objetos vistos não disponibilizam todas suas propriedades diretamente, os nossos olhos e nosso cérebro percebem coisas diferentes, ou melhor, as interpretações realizadas pelo nosso cérebro fazem com que os nossos olhos visualizem mais informações do que as fornecidas pelo objeto em si, as ações e as coordenações das ações do sujeito.

[...] se olharmos para os elementos do processo de visualização, os participantes são um sujeito que olha e um objeto que é olhado, no entanto, no que diz respeito à matemática, o que se olha nunca é o objeto que se quer saber, mas o signo que está em seu lugar, isto é, o signo matemático com propriedades e relações. (Soto, 2012, p. 23)

Assim, temos uma relação triangular entre: sujeito - objeto - signo, que estabelece o conhecimento do objeto, ou seja, estará dependente da participação desse signo, que são as propriedades do objeto as quais o sujeito deve apropriar-se para conhecer matematicamente o objeto de estudo, no momento dessa apropriação de conhecimento houve uma abstração reflexionante.

\section{0 potencial das tecnologias}

Qual o papel das tecnologias hoje no ensino da matemática? Segundo Kaput, Hegedus e Lesh (2007) as tecnologias se infiltram e transformam a matemática ao longo do tempo, acreditam em formas onipresentes da tecnologia nas escolas, melhor aprendizado, novo currículo e habilidades mais eficazes. Os autores utilizam o termo infraestrutura para o papel das tecnologias em relação à educação matemática. Lembrase que infraestrutura se refere à base, a instalações e serviços necessários.

Pesquisadores (Tall (1998), Notare e Basso (2015), Kaput, Hegedus e Lesh (2007)) estão sugerindo o uso dos softwares para transferir cálculos e operações, ou seja, transferir habilidades operacionais para as máquinas. Mas, diante disso, ainda não sabemos o que fazer sobre as novas habilidades que esse fato nos proporciona ou nos exige.

É necessário aprender a trabalhar com esses recursos, ou melhor, é preciso desenvolver uma matemática diferente com a parceria dessas ferramentas, que podem construir novas capacidades. Devemos pensar em um ensino de matemática que não poderia ser feito sem o uso das tecnologias. 
As habilidades desenvolvidas pelos sujeitos para a percepção visual (Gutierrez, 1996), tabela 1, são capacidades cognitivas não operatórias do nosso pensamento, ou seja, uma possibilidade de habilidades que podem proporcionar pensamentos matemáticos. $\mathrm{E}$ se tem uma variedade de recursos digitais que fornecem experiências com visualização.

Tabela 1 - Descrição das habilidades da visualização espacial

\begin{tabular}{|l|l|}
\hline \multicolumn{1}{|c|}{ Habilidade } & \multicolumn{1}{c|}{ Competência } \\
\hline Percepção figura - fundo & identificar uma figura isolando-a do fundo ou de outras \\
\hline Constância perceptiva & $\begin{array}{l}\text { reconhecer propriedades constantes de um objeto independente de seu } \\
\text { tamanho, orientação, cor }\end{array}$ \\
\hline Rotação mental & $\begin{array}{l}\text { produzir imagens mentais dinâmicas e visualizar características em } \\
\text { movimento }\end{array}$ \\
\hline $\begin{array}{l}\text { Percepção de posições } \\
\text { espaciais }\end{array}$ & relacionar um objeto, imagem ou imagem mental para si mesmo \\
\hline $\begin{array}{l}\text { Percepção de relações } \\
\text { espaciais }\end{array}$ & $\begin{array}{l}\text { relacionar vários objetos, imagens e imagens mentais para o outro, ou } \\
\text { simultaneamente para si mesmo }\end{array}$ \\
\hline Discriminação visual & $\begin{array}{l}\text { comparar objetos, imagens e imagens mentais para identificar semelhanças } \\
\text { ou diferenças entre eles }\end{array}$ \\
\hline
\end{tabular}

As tecnologias digitais de visualização e as interações entre sujeito e objetos geométricos proporcionadas por essas tecnologias podem contribuir para 0 desenvolvimento dessas habilidades.

Diante disso, verificou-se a necessidade dessa revisão sistemática da literatura para analisar se o uso das tecnologias está sendo utilizado para o desenvolvimento dessas habilidades geométricas espaciais. Ao que segue, descrevemos a metodologia utilizada para essa pesquisa.

\section{Procedimentos}

Uma revisão sistemática é uma técnica de pesquisa que segue uma metodologia bem definida com os passos documentados para que passe confiabilidade. Tem como objetivo identificar, avaliar e interpretar pesquisas relevantes sobre uma questão de pesquisa específica para identificar estudos futuros ou verificar o estado da arte para se desenvolver uma pesquisa relacionado à questão (Souza; Canalli, 2014).

É descrito a seguir as etapas e decisões tomadas para essa revisão. Primeiramente, verificamos a necessidade de realizar a revisão pois constatou-se, dos mesmos sistemas de busca que serão apresentados a seguir, que não há uma revisão sistemática que revisa as pesquisas em relação ao potencial das tecnologias para o desenvolvimento das habilidades geométricas espaciais.

Diante dos estudos apresentados nas seções anteriores, o foco é buscar estudos relacionados com a seguinte questão de pesquisa: Qual o panorama atual de pesquisas que envolvem o uso das tecnologias para o desenvolvimento do pensamento geométrico espacial no ensino básico?

Baseado nesta questão principal, outras questões específicas foram propostas para serem investigadas:

- Quais tecnologias digitais estão sendo aplicadas para o pensamento geométrico espacial?

- Como essas tecnologias estão sendo utilizadas?

- Quais habilidades da geometria espacial pretendeu-se desenvolver?

- Quais aportes teóricos foram utilizados para as análises?

Com essas questões, se buscou palavras-chaves que estivessem relacionadas com cada uma das questões, organizadas em três grandes áreas da questão principal, apresentadas na tabela que segue (escritas em português e inglês, já que foram utilizados 
banco de dados internacionais). A combinação dessas palavras e seus sinônimos formaram nossa string de busca.

Tabela 2 - Palavras para string de busca

\begin{tabular}{|c|c|c|}
\hline Área & \multicolumn{2}{|c|}{ Palavras e seus sinônimos } \\
\hline $\begin{array}{l}\text { Geometria } \\
\text { espacial }\end{array}$ & $\begin{array}{l}\text { "Geometria espacial" OU } \\
\text { Tridimensional OU Espaço }\end{array}$ & $\begin{array}{l}\text { "Spatial geometry" OR "Three- } \\
\text { dimensional" OR Space }\end{array}$ \\
\hline $\begin{array}{l}\text { Habilidades } \\
\text { cognitivas }\end{array}$ & $\begin{array}{l}\text { "Visualização espacial" OU } \\
\text { "Treinamento de habilidade espacial" } \\
\text { OU "Habilidades espaciais" OU } \\
\text { "Pensamento geométrico espacial" OU } \\
\text { "Percepção espacial" OU "Imaginação } \\
\text { espacial" }\end{array}$ & $\begin{array}{l}\text { "Spatial visualization" OR "Spatial ability } \\
\text { training" OR "Space skills" OR "Spatial } \\
\text { geometric thinking” OR "Spatial } \\
\text { perception" OR "Space imagination" }\end{array}$ \\
\hline Tecnologias & $\begin{array}{l}\text { "Software 3D" OU "Software de } \\
\text { modelagem 3D" OU "Geometria } \\
\text { dinâmica" OU "Objetos de } \\
\text { aprendizagem" OU Tecnologias OU } \\
\text { "Realidade aumentada" OU } \\
\text { "Impressão 3D" OU Estereoscopia OU } \\
\text { "Recursos de computador" }\end{array}$ & $\begin{array}{l}\text { "3d software" OR "3D modelling } \\
\text { software" OR "Dynamic geometry" OR } \\
\text { "Learning objects" OR Technologies OR } \\
\text { "Augmented reality" OR "3D printing" } \\
\text { OR Stereoscopy OR "Computer resources" }\end{array}$ \\
\hline
\end{tabular}

A definição dos parâmetros da pesquisa se restringiu a buscar periódicos, entre artigos e anais de eventos disponibilizados nos bancos de busca: Scopus, Springer, Web of Science, Scielo, e nesta própria revista, Renote. Inserindo as strings de busca foram encontradas 1475 pesquisas. Os critérios de inclusão foram que as pesquisas deveriam estar na língua inglesa, espanhola ou portuguesa e os textos deveriam estar disponíveis integralmente na rede online.

Os critérios de exclusão foram os seguintes: estudos explicitamente não relacionados ao tema (área da física, saúde, engenharia, arquitetura, mecânica), o público alvo não estar definido como sujeitos do ensino básico ou nessa faixa etária e trabalhos duplicados. Alguns desses critérios puderam ser aplicados no próprio sistema de busca e outros somente na leitura dos títulos, resumo ou até mesmo na leitura completa.

A seleção dos estudos foi um processo de quatro etapas: seleção do título, seleção pelo resumo, leitura completa e análise dos dados. Das 1475 pesquisas encontradas, após alguns critérios de inclusão/exclusão que se podia realizar pelo próprio sistema de busca, foi necessária a leitura de 349 resumos para chegarmos em 18 pesquisas que seguiram para a terceira etapa, a leitura completa da produção. Após a leitura completa das publicações, mais 9 artigos foram descartados seguindo os critérios de exclusão. Nas referências bibliográficas desse artigo é listado cada uma dessas publicações.

Na próxima seção é apresentada a quarta etapa da seleção: a análise desses artigos, levando em consideração a questão de pesquisa mencionada anteriormente, que norteou essa busca.

\section{Resultados e discussões em relação às pesquisas analisadas}

Após a exclusão dos trabalhos envolvendo alunos do ensino superior, por exemplo dos cursos de engenharia e arquitetura, onde enfatizam a importância da visualização e percepção espacial, é finalizado nossa busca na análise de nove pesquisas, ao que segue.

Huleihil (2017) utiliza a impressora 3D e o software CAD para construir sólidos, sendo esse software comerciável, mas muito utilizado por engenheiros, arquitetos, design 
e uma variedade de outros profissionais. O pesquisador acredita na metodologia STEM de ensino, método de ensino muito utilizada nos EUA, onde as disciplinas são integradas (Ciências, Tecnologia, Engenharia e Matemática) e também no ensino baseado em projetos. Sua proposta didática se destina a alunos do $6^{\circ}$ ano do Ensino Fundamental, mas na publicação desse artigo essa aplicação ainda não havia sido realizada. Essa proposta é constituída por três etapas: desenho no papel, desenho no CAD e posteriormente a impressão 3D, cada uma dessas etapas possui sub etapas onde habilidades são desenvolvidas e também conhecimentos de diferentes disciplinas são construídos. Diz que a impressão 3D na sala de aula motiva os estudantes, é capaz de elevar sua imaginação e ajuda os alunos a aumentar sua intuição espacial. Ainda, os alunos devem avaliar o custo de fabricação e entender a necessidade de otimização. O projeto em papel e os recursos e ferramentas dos sistemas CAD permitem ensinar conceitos e habilidades matemáticas através da construção e modelagem geométrica em duas e três dimensões. A impressão do objeto projetado possibilita a compreensão e a avaliação do projeto por meio da verificação do produto. Os conteúdos de geometria espacial desenvolvidos na sua experiência acontecem no desenho com projeções, diferentes vistas, perspectivas e no software CAD com o fornecimento dos resultados de área e volume dos sólidos construídos.

Outros pesquisadores, Bicer et al. (2017), também usam as tecnologias CAD e impressora 3D baseados na metodologia STEM de ensino, mas sua proposta difere da anterior, enfatiza muito o uso dessas tecnologias para despertar a criatividade e habilidades para a resolução de problemas. Bicer et al., propõe a 95 estudantes do ensino médio do Texas, em duas semanas de acampamento de verão, a projetarem um objeto físico, depois de orientados sobre o uso dessas tecnologias. Os autores enfatizam o desenvolvimento da criatividade e a habilidade de visualização, muito importantes para a resolução de problemas e necessário para as carreiras STEM, "É possível reduzir a carga na memória de trabalho dos alunos usando o software CAD e a impressão 3D para que eles possam dedicar mais capacidade intelectual à criação de ideias e produtos originais". Esses autores analisaram com mais detalhes os conteúdos contemplados com essa proposta, entre elas temos: escala, consciência espacial, medição, incluindo volume, área e dimensões lineares e vocabulário apropriado de matemática.

Em uma escola de Taiwan, num grupo de estudantes do $5^{\circ}$ ano, Sung, Shih e Chang (2014), aplicaram um sistema de ensino utilizando o software Google SketchUp para trabalhar com cálculo de área de sólidos compostos. Para suas análises utilizam préteste e pós-teste e comparação de um grupo controle, que recebeu instruções tradicionais, com um grupo experimental, que recebeu instruções pela tecnologia, envolvendo principalmente as múltiplas visualizações possibilitadas pelo uso do software, os autores chegaram em muitas conclusões, algumas já registradas por pesquisadores anteriores. Os objetivos do estudo eram: verificar se há diferença em desenvolvimento cognitivo com os alunos que receberam instruções pelo sistema digital, se há diferenças de habilidades em relação ao gênero dos estudantes, se há diferenças nas atitudes perante o estudo de matemática como uso da tecnologia e se os efeitos do sistema digital são moderados pelas habilidades ou gênero. Dizem que é muito difícil para os alunos converterem visões bidimensionais em tridimensionais, seguindo principalmente as ideias de Piaget e Van Hiele, os alunos do Ensino Fundamental encontram-se no período Operatório Concreto, precisam de características físicas para aprender, ou seja, se uma representação não é realmente concreta, os alunos estão propensos a ter dificuldades de aprendizagem. Em relação a composição de sólidos, salientam que o uso de material concreto traz benefícios limitados pois não abrange a variedade de composições possibilitadas pela tecnologia digital: "[...] manipular simplesmente os cubos físicos não é suficiente para que as 
crianças representem as formas do sólido básico a partir de diferentes perspectivas para estruturação espacial, nem pode ilustrar as relações entre cubos que constroem o sólido básico em diferentes vistas ortogonais para coordenação." (p. 118). As múltiplas representações do software contêm informações complementares para apoiar processos cognitivos, ou vincular essas representações para construir uma compreensão mais completa ou mais profunda de sólidos compostos.

Wahab (2017) também utilizou o software Google Sketchup, prepararam um material didático que elevasse as habilidades espaciais e os níveis de pensamento geométrico de Van Hiele, que consistem em quatro componentes: saber girar, visualizar, transformar e cortar mentalmente. $\mathrm{O}$ artigo analisado trata da validação desse material, que foi analisado por especialistas, dois alunos que avaliaram as instruções e posteriormente a aplicação com pré e pós-teste em 24 alunos. Perceberam no pós-teste que levaram menos tempo para solucionar as tarefas e ainda perceberam os alunos movimentando as mãos como se estivessem manipulando no software, além do melhor rendimento nas respostas.

Analisamos a seguinte pesquisa, onde os sujeitos não se enquadravam no nosso público, mas foram escolhidos aleatoriamente, ela não se destinava a um público específico, por isso não foi excluída pelos nossos critérios. Bach et al. (2018), comparam três tipos de tecnologia: realidade aumentada imersiva, realidade aumentada com smartphone e o uso de telas desktop tradicional em relação à percepção estereoscópica, liberdade para interação e proximidade dos espaços físicos para visualização. Utilizaram como teste nuvens de pontos e 15 sujeitos resolveram diversas atividades de percepção espacial. Foi analisado o tempo de conclusão das tarefas e também os erros. Chegaram à conclusão que o ambiente de desktop oferece precisão, velocidade e familiaridade superiores na maioria das tarefas que foram propostas.

Mui e Md-Ali (2017) da Malásia estudam a aplicação do guia "Guidelines for Using GeoGebra Dynamic Geometry Software in the Learning of Shape and Space", (Guia para os uso do Software de Matemática Dinâmica Geogebra na aprendizagem da forma e espaço) construído pelos próprios autores baseados na aprendizagem do Século 21, em que é necessário considerar as habilidades exigidas neste tempo, no baixo desempenho dos estudantes, principalmente em atividades que exigem aplicação dos conceitos em problemas, raciocínio e criatividade e também nos seus estudos sobre as potencialidades do software GeoGebra. Numa metodologia quasi-experimental, 102 alunos divididos em dois grupos experimentais e um controle com professores treinados pelos pesquisadores a aplicar o guia, chegam a conclusões mais focadas no despertar do interesse e envolvimento dos alunos com o uso das tecnologias, e não as contribuições desses para o desenvolvimento de habilidades cognitivas.

Clark e Ernst (2008) constroem um recurso tecnológico próprio para desenvolver habilidades visuais, o qual pretende que os alunos explorem conceitos com imagens estáticas ou dinâmicas. Os autores alegam que a capacidade dos estudantes em visualizar objetos tridimensionais rotacionados é essencial para entender e interpretar informações científicas, tecnológicas e matemáticas. Acreditam que com materiais baseados na visualização bem implementados podem contribuir com a melhoria das habilidades visuais. Para verificar a eficácia desse recurso, foi realizado como pré e pós-teste o Teste de Visualização Purdue, com 879 alunos de 14 professores que foram antecipadamente treinados. A faixa etária dos estudantes se encontrava entre 6 a 12 anos. Nestes testes verificou-se um aumento nas habilidades visuais, salientando essa melhora nos alunos do sexo masculino. Nas conclusões desse trabalho verificaram que são necessárias mais pesquisas sobre como desenvolver bons materiais com base na visualização, bem como aumentar a habilidade visual do aluno em todos os níveis e ainda, verificar a influência 
da mídia visual com a qual os alunos interagem diariamente para ver como as informações dessas mídias podem ser aproveitadas para melhorar as habilidades visuais.

Lowrie (2002) analisa como crianças pequenas se envolvem em ambientes de aprendizagem altamente visuais e relata um estudo que destaca as dificuldades de algumas crianças pequenas ao tentar interpretar imagens semelhantes a $3 \mathrm{D}$ em uma tela de computador. $\mathrm{O}$ autor relata que um software apropriado pode se tornar uma ferramenta poderosa onde as crianças podem manipular objetos espaciais e construir imagens visuais que normalmente seriam limitadas por suas capacidades de desenvolvimento. Doze crianças de seis anos foram convidadas a solucionar um problema que envolvia seus entendimentos espaciais na tela do computador, com esta atividade verificaram que as crianças apresentavam dificuldade de relacionar os ambientes 3D da tela do computador, ou seja, representados bidimensionalmente, em ambientes reais, com profundidade. Os autores relacionaram esses resultados com anteriores sobre o uso de fotografias. As ferramentas digitais disponibilizadas para essas crianças não permitiam a visualização, a rotação dos objetos, ou seja, eram imagens estáticas.

Publicado na revista Renote, Macedo, Silva e Buriol (2016), descrevem a criação e implementação de um aplicativo para smartphone com Realidade Aumentada (RA) que dá suporte para um material impresso também criado pelos autores sobre pirâmides, suas características, elementos, planificação, área e volume. As figuras do material impresso, podem ser projetadas em realidade aumentada, facilitando a visualização desse sólido. Os autores dizem que "[...] o fazer matemático através do uso das tecnologias deve provocar certa reflexão, e, sobretudo a organização consciente do conhecimento" (p. 2). Ainda afirmam que no mundo virtual podemos criar formas que até então só estavam disponíveis na nossa imaginação. Após aplicação do experimento, os autores concluíram que os alunos se mostraram receptivos quanto ao uso de dispositivos móveis, a RA contribuiu na motivação e interação dos alunos com relação ao conteúdo da aula e alguns alunos reconheceram como uma ferramenta eficiente na visualização de objetos 3D, e isso contribuiu para a aprendizagem do conteúdo abordado.

Percebe-se que é comum a todos os autores que as tecnologias digitais podem trazer benefícios para o ensino e aprendizagem da geometria espacial, principalmente a aspectos relacionados com a visualização de objetos tridimensionais. Há o uso de diferentes tecnologias: software de geometria dinâmica, software de modelagem 3D, impressora 3D, realidade virtual e aumentada, Google SketchUp e até aplicativos construídos pelos próprios pesquisadores, todos visando a visualização de objetos espaciais.

Algumas pesquisas salientaram mais a preocupação em relação a construção de conhecimentos, outros mostraram o uso como uma ferramenta puramente para aspectos visuais, também utilizado como um recurso motivacional para os estudantes e ainda como uma ferramenta desafiadora para produção de projetos e resolução de problemas contemplando outras habilidades.

\section{Considerações finais}

Com esta revisão sistemática percebeu-se que temos a disposição diferentes tecnologias que podem ser utilizadas em prol do pensamento geométrico, ou seja, recursos que podem desencadear ações dos sujeitos sobre objetos de estudo. Lembrando que Piaget (1981) salienta a importância das ações sobre os objetos para a construção do pensamento espacial.

Notou-se também que as potencialidades dessas tecnologias são vastas e que as pesquisas não esgotaram as possibilidades de uso em relação ao pensamento geométrico. 
Retomando a questão de pesquisa da revisão: Qual o panorama atual de pesquisas que envolvem o uso das tecnologias para o desenvolvimento do pensamento geométrico espacial no ensino básico? Se verificou uma carência de pesquisas divulgadas no cenário de publicações internacionais que contemplam e visam o desenvolvimento do pensamento geométrico com a tecnologia em estudantes do ensino básico.

Dessa forma, para próximos encaminhamentos, se tem a intenção de desenvolver uma pesquisa cujo objetivo é analisar como as tecnologias digitais, softwares de geometria dinâmica e o desenvolvimento de objetos próprios com a impressão 3D, proporcionam interações e experiências para desencadear abstrações reflexionantes visando o desenvolvimento do pensamento geométrico espacial.

Além disso, se almeja utilizar a potencialidade digital para pensamentos que seriam difíceis sem o uso dela, contemplar propostas didáticas para o pensar e não para habilidades operacionais que podem ser transferidas para as máquinas.

\section{Publicações analisadas na Revisão Sistemática:}

BACH, Benjamin; SICAT, Ronell; BEYER, Johanna; CORDEIL, Maxime; PFISTER, Hanspeter. The Hologram in My Hand: How Effective is Interactive Exploration of 3D Visualizations in Immersive Tangible Augmented Reality? IEEE Transactions on Visualization and Computer Graphics. v. 24, n. 1, jan. 2018

BICER, Ali Bicer; NITE, Sandra B.; CAPRARO, Robert M., BARROSO, Luciana R.; CAPRARO, Mary M.; LEE, Yujin. Moving from STEM to STEAM: The Effects of Informal STEM Learning on Students' Creativity and Problem Solving Skills with 3D Printing. IEEE, 2017.

CLARK, Aaron; ERNST, Jeremy. Visual Science and STEM-based 6-12 education. American Society for Engineering Education, 2008.

HULEIHIL, M. 3D printing technology as innovative tool for math and geometry teaching applications. IOP Conf. Series: Materials Science and Engineering, 2017

LOWRIE, Tom. The influence of visual and spatial reasoning in interpretingsimulated 3d worlds. International Journal of Computers for Mathematical Learning. n. 7, p. 301-318, 2002

MACEDO, Alex de Cassio; SILVA, João Assumpção da; BURIOL, Tiago Martinuzzi. Usando Smartphone e Realidade aumentada para estudar Geometria espacial. Revista Novas Tecnologias na Educação, Porto Alegre. v. 14, n. 2, dez. 2016.

MUI, Khor, K.; Ruzlan Md-Ali. Geogebra: Towards realizing 21st century learning in Mathematics education. Malaysian Journal of Learning and Instruction (MJLI), Special issue on Graduate Students Research on Education, 2017, p. 93-115.

SUNG, Yao-Ting, SHIH, Pao-Chen, CHANG, Kuo-En. The effects of 3Drepresentation instruction on composite-solid surface-area learning for elementary school students. Springer Science. n. 43, 2014.

WAHAB, Rohani Abd; ABDULLAH, Abdul Halim; MOKHTAR, Mahani; ATAN, Noor Azean; ABU, Mohd Salleh. Evaluation by Experts and Designated Users on the Learning Strategy using SketchUp Make for Elevating Visual Spatial Skills and 
Geometry Thinking. Revista Bolema, Rio Claro (SP). v. 31, n. 58, p. 819-840, ago. 2017.

\section{Referências Bibliográficas}

BASSO, Marcus; NOTARE, Márcia Rodrigues. Pensar-com Tecnologias Digitais de Matemática Dinâmica. Renote, Porto Alegre, v. 13, n. 2, 2015.

COLL, César; MONEREO, Carles (Orgs). Psicologia da Educação Virtual: aprender e ensinar com as tecnologias da Informação e da Comunicação. Porto Alegre: Artmed, 2010 .

GUTIÉRREZ, Angel. Procesos y habilidades em visualizacion espacial. Memorias del 3er Congreso Internac. Sobre Investig. Em Educ. Mat. Valencia, 1991

GUTIÉRREZ, Angel. Visualization in 3-Dimensional Geometry: In Search os a Framework. En L. Puig y A. Gutiérrez (Eds.), Proceedings of the 20th PME Conference, v. 1, p. 3 -9, 1996

KAPUT, Jim; HEGEDUS, Stephen; LESH, Richard. Technology Becoming Infrastructural in Mathematics Education. In: LESH, R.A; HAMILTON, E.; KAPUT, J. J. (Ed.). Foundations for the future in mathematics education. New Jersey: Lawrence Erlbaum Associates, 2007. p. 173-191

OLIVEIRA, Lívia de. A construção do espaço, segundo Jean Piaget. In: Revista Sociedade e Natureza. v. 17, n. 33. Uberlândia, dez. 2005. 105-117 p. Disponível em: < http://www.seer.ufu.br/index.php/sociedadenatureza/article/viewFile/9205/5667> Acesso em: 29/03/2018

PIAGET, Jean. Abstração reflexionante: Relações lógico-aritméticas e ordem das relações espaciais. Porto Alegre: Artes Médicas, [1977]1995.

PIAGET, Jean; INHELDER, Barbel. A representação do espaço na Criança. Trad. Bernardina Machado de Albuquerque. Porto Alegre: Artes Médicas, [1981]1993. 507 p.

SOTO, Claudia M. Acuña. La visualization como forma de ver em Matematicas: um acercamiento a la investigación. Editora Gedisa, España: 2012.

SOUZA, Letícia Capelão de; CANALLI, Hugo Leonardo. Relatório de Revisão

Sistemática da Literatura (SLR): Educação a distância, design e tecnologias assistivas para surdos: um panorama de 2007 a 2013, no estado de Minas Gerais. UFMG, 2014. 186 p. Disponível em < http://www.leticiacapelao.com/arquivos/academico/outras/CAPELAO-et-al-2014Relatorio-SLR-EAD-Design-TA-Surdos.pdf> . Acesso em: 17 abr. 2018.

TALL, David. Information Technology and Mathematics Education: Enthusiasms, Possibilities \& Realities. 1998. Disponível em:

<http://www.warwick.ac.uk/staff/David.Tall/downloads.html>. Acesso em: 16/03/2015.

TURGUT, Melih; UYGAN, Candas. Spatial ability training with 3D modelling software. 11th International Conference on Technology in Mathematics Teaching. Bari, July, 2013. Conference Proceedings, edited by Eleonora Faggiano and Antonella Montone. 